\title{
Die Versorgung der Civil Servants im Vereinigten Königreich
}

\author{
Martin Landauer
}

I. Grundlegung

1. Civil Service als Vergleichsgruppe

2. Rechtliche Stellung der Civil Servants

3. Alterssicherung der Civil Servants im Kontext allgemeiner sozialer Sicherung

II. Ausgestaltung der Alterssicherung der Civil Servants

1. State pensions
a) Basic State Pension
b) State Second Pension
c) Umwandlung von Renten in Zahlung einer Pauschalsumme

2. Betriebliche Altersversorgung
a) Allgemeines
b) Das Principle Civil Service Pension Scheme

3. Private Rentenkonten

a) Personal Pensions und Stakeholder Pensions

b) Partnership pension accounts

c) Civil Service Additional Voluntary Contribution Scheme

III. Bewertung 


\section{Grundlegung}

\section{Civil Service als Vergleichsgruppe}

Der öffentliche Dienst des Vereinigten Königreichs setzt sich aus verschiedenen Gruppen von Beschäftigten zusammen. Dem Civil Service 1 , der seit 1921 in den britischen Home Civil Service und den Northern Ireland Civil Service aufgespalten ist, ${ }^{2}$ kommt innerhalb dieser Gruppen aufgrund seiner zentralen Stellung im Staatsgefüge eine besondere Bedeutung zu. Im Civil Service waren zum 30.9.2007 ca. 532.000 Personen beschäftigt. ${ }^{3}$ Dies entspricht in etwa $10 \%$ der Beschäftigten des öffentlichen Sektors. ${ }^{4}$

Der Begriff „Civil Service“ geht ursprünglich auf die zivilen Angestellten der „East India Company" zurück, ${ }^{5}$ heute bilden die zivilen Bediensteten der Zentralregierung, der Ministerien mit übertragenen Befugnissen (devolved government departments) sowie deren executive agencies (von den Ministerien ausgelagerte Behörden, die in der Regel über keine eigenständige Rechtspersönlichkeit verfügen) seinen Hauptteil. ${ }^{6}$ Keine Angehörigen des Civil Service sind hingegen Richter, Inhaber politischer Ämter wie etwa Minister, Angestellte von Staatsbetrieben und des Nationalen Gesundheitsdienstes, Bedienstete der Lokalverwaltungen, der Polizei und der Universitäten oder das Lehrpersonal in öffentlichen Schulen. ${ }^{7}$ Auch die Bediensteten der ,Non-Departmental Public Bodies", von den Ministerien abgespaltene und nicht deren direkten Weisungen unterstellte öffentliche Stellen mit grundsätzlich eigener Rechtspersönlichkeit und eigenem Personal, haben bis auf wenige Ausnahmen nicht den Status von civil servants. ${ }^{8}$

Eine Unterscheidung der Beschäftigten des öffentlichen Dienstes in Beamten und sonstige Bedienstete wird nicht getroffen. Auch der Senior Civil Service, der aus den Spitzenpositionen innerhalb des Civil Service besteht, ${ }^{9}$ weist keine statusbezogenen

1 Zur Geschichte des Civil Service etwa Moses, The Civil Service of Great Britain, 1966, S. $19 \mathrm{ff}$.

2 Dieser Länderbericht wird im Folgenden primär auf den Home Civil Service eingehen und den nordirischen Civil Service nur dort erwähnen, wo Besonderheiten dies erfordern.

3 Dies entspricht in etwa 492.000 Vollzeitstellen, s. Office for National Statistics, Civil Service Statistics, 31 March $2008<$ http://www.statistics.gov.uk/pdfdir/cs0109.pdf> (Stand: 25.7.2009), S. 1.

4 Burnham/Pyper, Britain's Modernized Civil Service, 2008, S. 20.

5 S. etwa Chapman, The Higher Civil Service in Britain, 1970, S. 9.

$6 \mathrm{Zu}$ den Schwierigkeiten einer präzisen Definition des Begriffs in seiner heutigen Bedeutung etwa Burnham/Pyper, Civil Service (Fußn. 4), S. 19 f.

7 Vgl. Greenwood/Pyper/Wilson, New Public Administration in Britain, 3. Aufl. 2002, S. 73 f.

8 S. Cabinet Office, Public Bodies: A Guide for Departments, Chapter 5: Public Body Staff, 2007, $<\mathrm{http}: / /$ www.civilservice.gov.uk/Assets/5_public_body_staffv2_tcm6-2484.pdf> (Stand: 25.7.2009), para. 1.3.

9 Mit ca. 4.670 Bediensteten zum 31.3.2008 entfielen auf den Senior Civil Service weniger als $1 \%$ der Beschäftigten des gesamten Civil Service, s. Office for National Statistics, Civil Service Statistics, 31 March 2008 (Fußn. 3), Tabelle 1 (Anhang). 
Besonderheiten auf, die es rechtfertigen würden, die senior civil servants als Beamten zu betrachten. Nichtsdestotrotz stellt dieser Länderbericht auf den Civil Service als relevante Vergleichsgruppe zu Beamten im statusrechtlichen Sinn ab. Dieser Vergleichsgruppenbildung liegen folgende Erwägungen zugrunde: Bei den civil servants handelt es sich im Gegensatz zu den Trägern politischer Ämter ${ }^{10}$ jedenfalls traditionell um Amtspersonen, die eine dauerhafte berufliche Laufbahn im Staatsdienst verfolgen (,permanent career officials"). ${ }^{11}$ Insbesondere aber üben die civil servants funktionell betrachtet hoheitliche Tätigkeiten aus. Ihrer daraus resultierenden besonderen Bedeutung für das Staatsgefüge des Vereinigten Königreichs entspricht es, daß der Zugang zum Civil Service grundsätzlich den Staatsangehörigen bestimmter Nationen - in einigen Bereichen auch allein Staatsangehörigen des Vereinigten Königreichs - vorbehalten ist. ${ }^{12}$ Zudem stehen die civil servants - wie die folgende Beschreibung ihrer rechtlichen Stellung zeigen wird - in einem besonderen Näheverhältnis zur Krone.

\section{Rechtliche Stellung der Civil Servants}

Nach der Verfassung sind die civil servants Bedienstete der Krone. Ihre rechtliche Stellung ist im Einzelnen verfassungsrechtlich jedoch nicht vorgegeben. Auch existiert bislang kein eigenständiger gesetzlicher Rahmen, der die Grundlagen des Civil Service regeln würde. Die Stellung der civil servants im Staatsgefüge sowie ihre Rechte und Pflichten ergeben sich vielmehr aus einer Reihe verschiedenster Gesetze, Codes und tradierter Verfahrensweisen. ${ }^{13}$ Die Leitung des Civil Service unterfällt der Prärogative der Krone, ${ }^{14}$ wobei die Civil Service Order in Council $1995^{15}$ weitreichende Exekutivbefugnisse auf den Minister for the Civil Service übertrug. ${ }^{16}$ Gestützt auf diese Befugnisse wurden zwei Kodices mit besonderer Relevanz für die Rechtsstellung der civil

$10 \mathrm{Zu}$ deren Amtsdauer schon Scheid, Die rechtliche Stellung der englischen Staatsbeamten, 1914, S. $27 \mathrm{f}$.

11 Hierzu sowie zur Aufweichung der traditionellen Hauptmerkmale des Civil Service s. Greenwood/Pyper/Wilson, New Public Administration (Fußn. 7), S. 74 ff.

12 Vgl. Cabinet Office, Civil Service Nationality Rules, Guidance on Checking Eligibility, November $2007<\mathrm{http}: / /$ www.civilservice.gov.uk/Assets/November_2007_Guidance_tcm6-2456.pdf> (Stand: 25.7.2009). Bestimmte Positionen im Civil Service, die die nationale Sicherheit betreffen, sind allein Staatsangehörigen des Vereinigten Königreichs vorbehalten.

13 Burnham/Pyper, Civil Service (Fußn. 4), S. 5.

14 Näher Fredman/Morris, The State as Employer, 1989, S. 23 ff.; s. auch Burnham/Pyper, Civil Service (Fußn. 4), S. 9.

15 Civil Service Order in Council 1995 vom 15.3.1995, zuletzt geändert durch die Civil Service (Amendment) (No. 2) Order in Council 2008.

16 Der Civil Service (Management Functions) Act 1992 erlaubt es den Ministern allerdings, Leitungsfunktionen im Hinblick auf Personalangelegenheiten auf andere Staatsbedienstete unterhalb der Ministerebene zu delegieren. 
servants erlassen: erstens der Civil Service Management Code ${ }^{17}$ (CSMC), der sich an die Ministerien und deren executive agencies wendet und die Grundsätze zur Einstellung von civil servants, deren Beförderung, Versetzung, Verrentung und Entlassung sowie das von ihnen zu erwartende Verhalten regelt; zweitens der Civil Service Code $(\mathrm{CSC})^{18}$, der ebenfalls Verhaltenspflichten der civil servants festlegt, sich jedoch unmittelbar an diese selbst richtet und Bestandteil ihrer vertraglichen Beziehungen zum Arbeitgeber ist. 19

Aus den genannten Quellen lassen sich einige Charakteristika des Civil Service ableiten: Die Einstellung in den Civil Service basiert auf dem Verdienstprinzip ${ }^{20}$ und stellt auf Eignung und Qualifikation der Bewerber ab. Die Anstellung beruht jedenfalls im Senior Civil Service ${ }^{21}$ und nach vorzugswürdiger Ansicht auch bei den übrigen civil servants auf vertraglicher Basis, ${ }^{22}$ eine Ernennung durch Urkunde findet nicht statt.

Den Civil Service kennzeichnet zwar die grundsätzlich dauerhafte Anstellung seiner Bediensteten, dieser Aspekt bezieht sich aber primär auf die Personalkontinuität im Falle eines politischen Wechsels an der Spitze der Ministerien. ${ }^{23}$ Insofern verfügt der Civil Service durchaus über Bedienstete mit befristeten Verträgen, ${ }^{24}$ und auch der Schutz vor Entlassungen ist - jedenfalls aus rechtlicher Sicht - weniger stark ausgeprägt als bei anderen Arbeitnehmern: Zwar finden die Vorschriften des Employment Rights Act

17 Cabinet Office, Civil Service Management Code, $2008<\mathrm{http}: / /$ www.civilservice.gov.uk/ about/work/codes/csmc/index.aspx> (Stand: 25.7.2009).

18 Cabinet Office, Civil Service Code, $2006<$ http://www.civilservice.gov.uk/Assets/cs_code_tcm62444.pdf> (Stand: 25.7.2009); für die civil servants von Nordirland gilt ein eigener Kodex, der sich inhaltlich mit dem CSC weitgehend deckt, vgl. Northern Ireland Civil Service, Northern Ireland Civil Service Code of Ethics, $2006<\mathrm{http}$ ://www.nicscommissioners.org/documents/nics-code-ofethics.pdf $>$ (Stand: 25.7.2009). Allgemein zu Struktur und rechtlicher Stellung des Northern Ireland Civil Service etwa Carmichael, The Northern Ireland Civil Service: Characteristics and Trends since 1970, Public Administration 80 (2002), S. 23 ff. Im Folgenden werden, soweit der Northern Ireland Civil Service Code of Ethics keine Besonderheiten aufweist, allein die Vorschriften des CSC zitiert.

19 Cabinet Office, Civil Service Code (Fußn. 18), para. 19.

20 Siehe etwa Civil Service Order in Council 1995, Article 2; CSC (Fußn. 18), para. 2; dieses Prinzip geht zurück auf den Bericht von Northcote/Trevelyan, Report on the Organisation of the Permanent Civil Service, 1854; hierzu und zur Entwicklung des Einstellungsverfahrens Chapman, Higher Civil Service (Fußn. 5), S. 23 ff., 37 ff.; zum aktuellen recruitment s. Burnham/Pyper, Civil Service (Fußn. 4), S. 193 ff.

21 Vgl. Cabinet Office, Civil Service Management Code (Fußn. 17), para. 5.3.2. Der Mustervertrag für den Senior Civil Service ist abrufbar unter $<$ http://www.civilservice.gov.uk/Assets/STANDARD\% 20June\%2006_tcm6-2429.doc> (Stand: 25.7.2009).

22 Vgl. etwa R v Civil Service Appeal Board ex parte Bruce [1988] 3 All E.R. 686; McClaren v Home Office [1990] I.C.R. 824; dazu auch Craig, Administrative Law, 5. Auflage 2003, S. 162 f.; Grunfeld, Civil Servants: Insecurity of Tenure, MLR 23 (1960), S. 194, 195 f.; für diese Ansicht spricht im übrigen auch der Wortlaut des CSC, vgl. Cabinet Office, Civil Service Code (Fußn. 18), para. 19; vgl. zu dieser Frage auch Fredman/Morris, The State (Fußn. 14), S. 61 ff.

23 Dazu etwa Burnham/Pyper, Civil Service (Fußn. 4), S. 13.

$24 \mathrm{Zu}$ den temporary civil servants vgl. Burnham/Pyper, Civil Service (Fußn. 4), S. $193 \mathrm{ff}$. 
$1996^{25}$ über den Schutz vor unfairen Entlassungen mittlerweile auch auf den Civil Service Anwendung, ${ }^{26}$ jedoch gilt es als Recht der Krone, den civil servant grundsätzlich jederzeit fristlos entlassen zu können (dismissal at will). ${ }^{27}$ In der Praxis genießen die civil servants allerdings eher hohe Arbeitsplatzsicherheit, 28 die Einhaltung von Kündigungsfristen ist üblich, und bei Nichteinhaltung werden Entschädigungszahlungen fällig. ${ }^{29}$

Die civil servants sind der Krone zu Loyalität und Verschwiegenheit verpflichtet ${ }^{30}$ und haben sich zu einem gewissen Grad politisch neutral zu verhalten. ${ }^{31}$ Ferner wird von den civil servants Integrität in dem Sinne erwartet, daß sie ihre persönlichen Interessen den Verpflichtungen des öffentlichen Dienstes unterordnen. ${ }^{32}$

Der Bezahlung der civil servants liegt kein Alimentationsprinzip zugrunde, das der Sicherung eines angemessenen Lebensunterhalts dienen würde. Die Maßstäbe für ihre Vergütung orientierten sich - mit einer im Laufe der Zeit oftmals wechselnder Gewichtung - einerseits an Forderungen, daß sie lediglich die Versorgung des Civil Service mit effizient arbeitendem Personal zu gewährleisten hätten, und andererseits an dem Postulat, der Staat als ,model employer" solle ein faires Gehaltsniveau bieten. ${ }^{33}$ Die vormals einheitlichen Gehaltsregelungen des Civil Service sind inzwischen einem Gehaltsschema für den Senior Civil Service und einer Vielzahl von Sonderregimes für die übrigen civil servants gewichen. ${ }^{34}$

Im Zusammenhang mit den hier aufgezeigten Grundsätzen ist allerdings zu bedenken, daß die durch die Regierung ausgeübte Prärogative der Krone zur Leitung des Civil Service es bislang ermöglicht, den Civil Service jederzeit in weiten Bereichen ohne Einbeziehung des Parlaments zu reformieren. Begrenzt werden diese Möglichkeiten in der Praxis derzeit vor allem durch den politischen Einfluß der Gewerkschaften. ${ }^{35}$

251996 Chapter 18.

26 Employment Rights Act 1996, section 191(1), (2)(e) i.V.m. sections 94 ff.

27 Employment Rights Act 1996, section 191(1), (2)(d) verweist gerade nicht auf die für die Kündigungsfrist relevanten sections 86 ff.; zum Recht der Krone zur fristlosen Kündigung vgl. auch Dunn v Queen [1896] 1 QB 116. Das case law deutet darauf hin, daß dieses Recht durch Vertrag ausgeschlossen werden kann, vgl. Robertson v Minister of Pensions [1949] 1 K.B. 227, 231; anders allerdings Rodwell v Thomas and Others [1944] K.B. 596; dazu Craig, Administrative Law (Fußn. 22), S. 164; Fredman/Morris, The State (Fußn. 14), S. 68 ff.

28 Fredman/Morris, The State (Fußn. 14), S. 68.

29 Vgl. etwa den Mustervertrag für den Senior Civil Service (Fußn. 21), section 14.

30 Cabinet Office, Civil Service Management Code (Fußn. 17), para 4.1.1. sowie section 4.2.

31 Cabinet Office, Civil Service Code (Fußn. 18), paras. 13 f.; Cabinet Office, Civil Service Management Code (Fußn. 17), section 4.4.

32 Cabinet Office, Civil Service Code (Fußn. 18), para. 2; s. auch Richards, New Labour and the Civil Service, 2008, S. $16 \mathrm{ff}$.

33 Fry, The Changing Civil Service, 1985, S. 96 ff.; vgl. auch Fredman/Morris, The State (Fußn. 14), S. 190 ff.; Burnham/Pyper, Civil Service (Fußn. 4), S. 214 ff.

34 Näher Burnham/Pyper, Civil Service (Fußn. 4), S. 210 ff.

35 Vgl. Burnham/Pyper, Civil Service (Fußn. 4), S. 12, 192 f., 215 f. 
Die Zukunft läßt allerdings eine stärkere gesetzliche Fundierung des Civil Service erwarten. Der Lord Chancellor, Jack Straw, legte dem Parlament am 25.03.2008 eine Draft Constitutional Renewal Bill vor, ${ }^{36}$ die im 5. Teil in 17 Paragraphen einen rechtlichen Rahmen für die Werte und die Leitung des britischen ${ }^{37}$ Civil Service entwirft. Dieser Entwurf, der inzwischen auch die grundsätzliche Zustimmung des Joint Committee on the Draft Constitutional Renewal Bill des House of Lords und des House of Commons gefunden hat, ${ }^{38}$ stellt unter anderem die Leitungsbefugnis des Minister for the Civil Service sowie den CSC auf eine gesetzliche Basis und verlangt, die jeweils gütige Fassung des CSC dem Parlament vorzulegen. ${ }^{39}$ Mittlerweile wurde die Constitutional Renewal Bill als sog. Privat Members Bill in das House of Lords eingebracht und dort in erster Lesung behandelt; 40 die den Civil Service betreffenden Regelungen befinden sich nun - in gegenüber der Draft Bill im Wesentlichen unveränderter Form - im 4. Teil in den sections $25 \mathrm{ff}$. Inhaltliche Änderungen an den genannten Charakteristika des Civil Service lassen sich der Constitutional Renewal Bill jedoch nicht entnehmen.

\section{Alterssicherung der Civil Servants im Kontext allgemeiner sozialer Sicherung}

Historisch bildeten die britischen civil servants die erste Gruppe von Beschäftigten, für die der Staat mit einem Act of Parliament des Jahres $1810^{41}$ und dem Superannuation Act 183442 ein umfassendes Rentenversicherungssystem geschaffen hatte. ${ }^{43}$ Aus diesen Anfängen hat sich - maßgeblich beeinflußt durch den Old Age Pensions Act $1908^{44}$ und die Sozialgesetzgebung in Folge des Beveridge Reports ${ }^{45}$ - das heutige Rentenversicherungssystem entwickelt. ${ }^{46}$ Es setzt sich aus der als Grundsicherung gedachten universalen staatlichen Vorsorge, der betrieblichen Altersvorsorge und privaten

36 Ministry of Justice, The Governance of Britain - Draft Constitutional Renewal Bill, Cm 7342-II, März 2008.

37 Nach Ministry of Justice, Draft Constitutional Renewal Bill (Fußn. 36), section 25(2), sollen die Regelungen auf den Civil Service Nordirlands keine Anwendung finden.

38 House of Lords/House of Commons - Joint Committee on the Draft Constitutional Renewal Bill, Draft Constitutional Renewal Bill, Volume I: Report, HL Paper 166-I, HC Paper 551-I, 31.7.2008, S. $67 \mathrm{ff}$.

39 Ministry of Justice, Draft Constitutional Renewal Bill (Fußn. 36), sections 27, 30.

40 Constitutional Renewal Bill [HL] 2008-09 (HL Bill 34 08-09).

4150 George III, Chapter 117.

421834 Chapter 24.

43 Dazu Blake, Pension Schemes and Pension Funds in the United Kingdom, 2. Aufl. 2003, S. 3 ff.

441908 Chapter 40.

45 Beveridge, Social Insurance and Allied Social Services, Cm 6406, 1942.

46 Zur historischen Entwicklung des Rentenversicherungssystems s. Blake, Pension Schemes (Fußn. 43), S. 3 ff., 22 ff.; Hamisch, Der Schutz individueller Rechte bei Rentenreformen, 2001, S. 55 ff.; Kaempfe, Die Systemfunktionen privater Altersvorsorge im Gesamtsystem sozialer Alterssicherung, 2003, S. $103 \mathrm{ff}$. 
Vorsorgeplänen zusammen. In dieses allgemeine System sind mit einigen Besonderheiten auch die civil servants einbezogen.

\section{Ausgestaltung der Alterssicherung der Civil Servants}

\section{State pensions}

\section{a) Basic State Pension}

Das erste Element der staatlichen Vorsorge bildet die Basic State Pension (BSP). ${ }^{47}$ In diesem System sind alle Erwerbstätigen - und somit auch die civil servants - pflichtversichert. Die BSP stellt eine von der Höhe der gezahlten Beiträge unabhängige Einheitsrente dar, die derzeit ab einem Rentenalter von 65 Jahren (bei Männern) bzw. 60 Jahren (bei Frauen) bezahlt wird. Das Rentenalter der BSP wird bis zum Jahr 2020 schrittweise auch für Frauen auf 65 und danach bis 2046 für beide Geschlechter auf 68 Jahre angehoben werden. ${ }^{48}$ Wenn die BSP in voller Höhe bezogen werden kann, wofür bei Männern aktuell 44 und bei Frauen 39 anspruchsbegründende Beitragsjahre erforderlich sind, ${ }^{49}$ beträgt sie in Gestalt der primär einschlägigen Rente der Kategorie A derzeit wöchentlich $£$ 95.25. ${ }^{50}$ Die Rentenhöhe wurde bislang zum April eines jeden Jahres an die Inflationsrate angepaßt, mittelfristig ist eine Koppelung an die Entwicklung des durchschnittlichen Lohnniveaus geplant. 51

Die BSP wird im Wege eines Umlageverfahrens hauptsächlich aus den Beiträgen zur National Insurance finanziert, die für alle Sozialversicherungszweige gemeinsam erhoben werden. Ca. 10-15 \% des finanziellen Gesamtaufwandes werden durch einen Staatszuschuß gedeckt. 52

47 Die wichtigsten rechtlichen Grundlagen für die BSP stellen der Social Security Contributions and Benefits Act 1992 (1992 Chapter 4) sowie der Pensions Act 1995 (1995 Chapter 26) dar; allgemein zur BSP etwa Rechmann, Altersicherung in der Europäischen Union VI, Alterssicherung in Großbritannien und Irland, 2001, S. $90 \mathrm{ff}$.

48 Vgl. Pensions Act 1995, section 126 (in der durch den Pensions Act 2007, section 13, geänderten Fassung).

49 Vgl. Social Security Contributions and Benefits Act 1992, section 44(1A) i.V.m. Schedule 3 para. 5(1), (2), (5). Die erforderlichen Beitragsjahre werden ab 2010 auf 30 reduziert werden, vgl. Security Contributions and Benefits Act 1992, section 44(1A) i.V.m. Schedule 3 para. 5A, außerdem wurde durch den Pensions Act 2007 bzw. den Pensions Act (Northern Ireland) 2008 in größerem Umfang als bisher die Möglichkeit geschaffen, anspruchsbegründende Beitragsjahre des Ehe- oder Lebenspartners zur Begründung einer eigenen Rente heranzuziehen.

50 Social Security Contributions and Benefits Act 1992, section 44(4).

51 The Pension Service, State Pensions - Your Guide, $2008<\mathrm{http}: / / \mathrm{www}$. thepensionservice.gov.uk/ $\mathrm{pdf} / \mathrm{pm} / \mathrm{pm} 2 \mathrm{jan} 09 . \mathrm{pdf}>$ (Stand: 25.7.2009), S. 35.

52 Hamisch, Schutz individueller Rechte (Fußn. 46), S. 64 f. 


\section{b) State Second Pension}

Die State Second Pension (S2P) bildet als Additional State Pension das zweite Element der staatlichen Rentenversicherung. Die S2P stellt eine eigenständige, beitragsfinanzierte Zusatzrente dar. Sie wurde durch den Child Support, Pensions and Social Security Act 200053 eingeführt und löste zum 6.4.2002 das State Earnings-Related Pension Scheme (SERPS) ${ }^{54} \mathrm{ab}$, um die Rentenkonditionen für Personen mit einem geringen bis mittleren Einkommen zu verbessern. ${ }^{55}$ Das Rentenalter entspricht dem der BSP. Ansprüche auf eine S2P können von Arbeitnehmern mit einem Einkommen von mehr als $£ 4.940$ pro Jahr erworben werden, wenn aus diesem Beiträge zur National Insuran$c e$ entrichtet werden. ${ }^{56}$ Die Rente basiert bislang zu einem gewissen Grad auf der Höhe des Einkommens und der geleisteten Beiträge. ${ }^{57}$ Hinsichtlich der Finanzierung und der Indexierung gelten die gleichen Grundsätze wie bei der BSP.

Wer über eine ausreichende betriebliche oder private Altersvorsorge verfügt, kann aus der S2P aussteigen (sog. contracting-out). ${ }^{58}$ Der ganz überwiegende Teil der civil servants ist in ein entsprechendes betriebliches oder privates Vorsorgesystem einbezogen, so daß die S2P für die Bediensteten des Civil Service praktisch keine Rolle spielt. 59

Aufgrund von Änderungen durch den Pensions Act 200760 kann die Möglichkeit zum contracting-out basierend auf einem defined contribution scheme künftig ab einem erst noch festzulegenden Zeitpunkt ${ }^{61}$ ausgeschlossen werden. ${ }^{62}$ Bei defined contribution schemes handelt es sich um Rentensysteme, bei denen die späteren Rentenleistungen von der Summe der eingezahlten Beiträge und der mit diesen erwirtschafteten Renditen abhängt. Dies bedeutet, daß ein contracting-out aus S2P künftig nur noch auf der

532000 Chapter 19, sections $30 \mathrm{ff}$.

54 SERPS war seinerseits auf das State Graduated Retirement Pension Scheme gefolgt. Nach wie vor werden Altrenten auf Basis beider Systeme geleistet.

55 Vgl. Blake, Pension Schemes (Fußn. 43), S. 80.

56 Vgl. The Social Security Contributions Regulations 2001, S.I. 2001, No. 1004, reg. 10, in der durch The Social Security (Contributions) (Amendment No. 2) Regulations 2009, S.I. 2009 No. 591 geänderten Fassung.

57 Der Einkommensabhängigkeit liegen zugunsten kleiner und mittlerer Einkommen allerdings gewisse Pauschalisierungen zugrunde. Derzeit werden beispielsweise Renten bei Personen mit einem Einkommen zwischen $£ 4.940$ und $£ 13.900$ (vgl. The Social Security Pensions (Low Earnings Threshold) Order 2009, S.I. 2009 No. 610, art. 2) auf Basis eines (fiktiven) Jahreseinkommens von $£ 13.900$ berechnet. Aufgrund des Pensions Act 2007 wird die Einkommensabhängigkeit der Rentenhöhe von 2010 weiter reduziert werden, bis 2030 soll sie schließlich ganz entfallen sein. Der Berechnung liegt eine komplexe Rentenformel zugrunde, vgl. Social Security Contributions and Benefits Act 1992, section 45(2).

$58 \mathrm{Zu}$ den Voraussetzungen siehe Pension Schemes Act 1993 Chapter 48, sections 9 ff.

59 Dazu unten, S. 224, insb. Fußn. 92.

602007 Chapter 22.

61 Als wahrscheinlicher Zeitpunkt gilt derzeit der 6.4.2012.

62 Pensions Act 2007, section 15. 
Grundlage von defined benefit schemes vorgenommen werden kann, d.h. nur noch basierend auf solchen Rentenplänen, die aufgrund einer festen Rentenformel (die üblicherweise auf dem bezogenen Gehalt und der Zahl der Versicherungsjahre beruht) bestimmte Rentenleistungen garantieren.

\section{c) Umwandlung von Renten in Zahlung einer Pauschalsumme}

Eine Pauschalsumme (lump sum) kann nur unter sehr engen Voraussetzungen anstelle einer staatlichen Altersrente bezogen werden, nämlich dann, wenn der Bezug der Rente nach Erreichen des Rentenalters aufgeschoben wird. Die Pauschalzahlung bezieht sich dann aber lediglich auf den aufgeschobenen Teil der Rente, der in einer gewissen Höhe verzinst wird. ${ }^{63}$ Sie ersetzt nicht die zukünftigen Rentenzahlungen.

\section{Betriebliche Altersversorgung}

\section{a) Allgemeines}

Die betrieblichen Altersversorgungssysteme (occupational pension schemes) bilden die 2. Säule des Rentenversicherungssystems. ${ }^{64}$ Sie sind jeweils mit dem Arbeitsverhältnis zu einem bestimmten Arbeitgeber verbunden und stellen im Hinblick auf die Rentenleistungen in der Regel als defined benefit schemes auf die Gehaltsentwicklung während des gesamten Erwerbslebens oder auf das Gehaltsniveau kurz vor Eintritt in das Rentenalter (final salary) ab. Seit wenigen Jahren finden sich allerdings auch defined contribution schemes oder gemischte Formen von Betriebsrenten. ${ }^{65}$ Die Schaffung eines occupational pension schemes geschieht auf freiwilliger Basis und kann zum contracting-out aus S2P berechtigen. ${ }^{66}$ Die Konditionen der betrieblichen Altersversorgung sind im öffentlichen Sektor generell attraktiver als im privaten Sektor. ${ }^{67} 2007$ waren im Vereinigten Königreich 3,6 Millionen Arbeitnehmer des privaten Sektors und 5,2 Millionen des öffentlichen Sektors aktive Mitglieder eines betrieblichen Altersvorsorgesystems. ${ }^{68}$

63 Vgl. Social Security Contributions and Benefits Act 1992, section 55 i.V.m. Schedule 5.

64 Ausführlich zu den occupational pensions etwa Blömeke, Die Regulierung nicht-staatlicher Alterssicherung, 2007, S. $57 \mathrm{ff}$.

65 Blake, Pension Schemes (Fußn. 43), S. 94.

66 Vgl. Pension Schemes Act 1993, sections 9 ff., 12A ff.

67 Record, Sir Humphrey's Legacy, 2006, S. 120.

68 Office for National Statistics, Occupational pension schemes annual report 2007, $2008<\mathrm{http} / / /$ www.statistics.gov.uk/downloads/theme_population/Occ-Pension-2007/OPSS_Annual_Report_200 7.pdf> (Stand: 25.7.2009), S. 16 . 
In Reaktion vor allem auf den Skandal um Robert Maxwell, bei dem zu Beginn der 1990er Jahre Arbeitnehmer um Betriebsrenten in Höhe mehrerer hundert Millionen Pfund gebracht worden waren, ${ }^{69}$ erließ das Parlament mit dem Pensions Act $1995^{70}$ strenge und umfassende Regelungen für die organisatorische Ausgestaltung und die Verwaltung der betrieblichen Altersversorgung. Gleichzeitig wurde die Occupational Pensions Regulatory Agency als Regulierungsbehörde für die Rentenversicherungsindustrie geschaffen. Sie wurde 2005 durch The Pensions Regulator ersetzt. ${ }^{71}$ Ferner existiert mit dem Pension Protection Fund 72 ein Sicherungsfonds, der im Falle der Insolvenz des Arbeitgebers Kompensationszahlungen an die Mitglieder von defined benefit schemes zahlt. 73

\section{b) Das Principle Civil Service Pension Scheme}

Beim occuppational scheme des Civil Service, dem sog. Principal Civil Service Pension Scheme (PCSPS) besteht die Besonderheit, daß es auf gesetzlicher Grundlage beruht. ${ }^{74}$ Zum 1.10.2002 wurde das PCSPS in drei verschiedene Versorgungspläne aufgeteilt. 75 Das bisherige System bekam - ohne wesentliche inhaltliche Änderungen - den Namen classic und sah weiterhin Rentenleistungen basierend auf einer Rentenquote von 1/80 pro Dienstjahr sowie eine steuerfreie Einmalzahlung in dreifacher Höhe der Jahresrente vor. Alternativ war der sog. premium Tarif wählbar, dessen Rentenquote bei 1/60 pro Dienstjahr lag. Die Renten beider Tarife können gegenwärtig bis zu einer Höhe von maximal 30/7 der Jahresrente gegen eine (in Falle von classic zusätzliche) steuerfreie lump sum von $£ 12$ für jedes $£ 1$ jährlich aufgegebener Pension getauscht werden. Classic plus als dritte Tarifoption bildete schließlich eine Kombination beider vorgenannter Modelle. ${ }^{76}$ Allen schemes lag das final salary Prinzip zugrunde. ${ }^{77}$

69 Ausführlich Blake, Pension Schemes (Fußn. 43), S. 340 ff.

701995 Chapter 26.

71 Pensions Act 2004 Chapter 35, section 1. Zu deren Aufgaben vgl. etwa Blömeke, Regulierung (Fußn. 64), S. 63 f.

72 Der Pension Protection Fund ist ein gesetzlicher Sicherungsfonds, der vom Board of the Pension Protection Fund, einer durch den Pensions Act 2004 errichteten Institution, getragen wird.

73 Näher zur Insolvenzsicherung Blömeke, Regulierung (Fußn. 64), S. 71 ff.

74 Die Grundlage bildet - zurückgehend auf den Superannuation Act 1834 - derzeit der Superannuation Act 1972 Chapter 11.

75 Dazu Rothenbacher, The Welfare State of the Civil (or Public) Servants in Europe: A Comparison of the Pension Schemes for Civil (or Public) Servants in France, Great Britain, and Germany, 2004, S. 48.

76 Für zwischen 1.10.2002 und 29.7.2007 eingestellte Bedienstete gelten diese drei Tarife fort.

77 Die Renten waren grundsätzlich gekoppelt an das Gehalt entweder der letzten zwölf Monate vor Pensionierung oder des besten der letzten vier Jahre vor Pensionierung oder des besten durchschnittlichen 3-Jahres-Zeitraums innerhalb der letzten 13 Jahre, je nachdem, woraus sich die höhere Basis ergab. Vgl. Principal Civil Service Pension Scheme 1974, Section I (The 2002 Section), rule A.4. 
Seit dem 30. Juli 2007 gilt für neu eingestellte civil servants ein neues betriebliches Versorgungssystem namens nuvos, ${ }^{78}$ das unter Beteiligung der Gewerkschaften entwickelt wurde und in seiner endgültigen Form auf große Zustimmung bei den Gewerkschaftsmitgliedern stieß.79 In nuvos, einem defined benefit scheme, erwerben die civil servants mit jedem Dienstjahr 2,3\% des im betreffenden Jahr erzielten pensionsrelevanten Gehalts (pensionable earnings) ${ }^{80}$ als Basis für die spätere Rentenleistung. ${ }^{81}$ Die gegenüber classic (1/80) und premium (1/60) deutlich erhöhte jährliche Quote (von 2,3 \%, also in etwa 1/43) dient als Ausgleich für die Aufgabe des final salary Prinzips zugunsten einer auf die gesamte Erwerbsbiographie bezogenen Basis. ${ }^{82}$ Man sah in der Tatsache, daß civil servants ihre Rentenbezüge durch eine Verlängerung der Arbeitszeiten im Referenzzeitraum nach oben treiben konnten, nicht nur eine erhöhte Ausgabenbelastung des Systems, sondern erachtete das Abstellen auf die letzten Dienstbezüge auch als Ungleichbehandlung derer, die den Civil Service frühzeitig verließen. ${ }^{83}$

Der Wert der erworbenen Pensionsteile wird sowohl vor als auch während des Bezugs der Rente jährlich an die Preisentwicklung angepaßt. ${ }^{84}$ Das Rentenalter wurde mit der Umstellung auf nuvos von 60 auf 65 Jahre angehoben, ab Vollendung des 55. Lebensjahres ist eine Frühverrentung unter Abschlägen möglich. ${ }^{85}$ Der letzte mögliche Zeitpunkt des Rentenbeginns liegt bei Vollendung des 75. Lebensjahres. ${ }^{86}$

Auch in nuvos kann ein Teil der Leistungen steuerfrei als lump sum bezogen werden. Jeweils $£ 1$ der jährlichen Rente kann in $£ 12$ Pauschalzahlung umgewandelt werden.

78 Die rechtlichen Regelungen für nuvos sind im Principal Civil Service Pension Scheme 1974, Section III (The 2007 Section) enthalten, das aufgrund des Superannuation Act 1972 erlassen wurde.

79 Vgl. etwa Public and Commercial Services Union, Annual Report 2007 <http://www.pcs.org.uk/ download.cfm?docid=C8BAF9B3-9B9A-4801-A3A20BC364F1D8AD $>$ (Stand: 25.7.2009), S. 7.

$80 \mathrm{Zu}$ den pensionable earnings zählen vereinfacht gesprochen alle dauerhaften Bestandteile der Einkünfte aus dem Dienstverhältnis, auch wenn es sich um Sachbezüge wie Uniformen oder Dienstwohnungen handelt. Zahlungen für Überstunden oder Boni sind grundsätzlich nicht relevant. Eine Obergrenze für das pensionsrelevante Gehalt existiert in nuvos im Hinblick auf die Bemessung der Beiträge nicht.

81 Principal Civil Service Pension Scheme 1974, Section III (The 2007 Section), rule C.2 para. (1). Die Pension darf grundsätzlich aber nicht mehr als 75\% des zuletzt erzielten Einkommens betragen, Principal Civil Service Pension Scheme 1974, Section III (The 2007 Section), rule A.6. Dies wird jedoch allenfalls bei sehr langen Dienstzeiten relevant. Neben Rentenleistungen für den civil servant sieht nuvos diverse Versorgungsleistungen zugunsten Hinterbliebener vor.

82 Für Arbeitnehmer, die ab dem 30.7.2008 nach einer Unterbrechung in den Civil Service dorthin zurückkehren oder die von einem anderen Arbeitgeber des öffentlichen Dienstes mit einem final salary related scheme in den Civil Service wechseln, wird die alte Rente in das PCSPS transferiert, dort aber unter Beibehaltung des Bezugs zum letzten Gehalt als separater Rententeil geführt.

83 Hansard, 26.7.2007, Col 105WS

84 Principal Civil Service Pension Scheme 1974, Section III (The 2007 Section), rule C.2 para. (8).

85 Principal Civil Service Pension Scheme 1974, Section III (The 2007 Section), rule A.1 para. (4). Ein vorzeitiger Rentenbezug zu speziellen Bedingungen ist möglich, wenn der civil servant aus gesundheitlichen Gründen aus dem Dienst ausscheiden mußte.

86 Vgl. Principal Civil Service Pension Scheme 1974, Section III (The 2007 Section), rule E.1 para. (1). 
Die Pauschalsumme kann maximal 30/7 der jährlichen Rente (vor Umwandlung) betragen. ${ }^{87}$ Der classic Tarif sah die Zahlung der lump sum in Höhe der 3-fachen Jahresrente noch als Regelfall an. Wer die Pauschalsumme unter classic nicht in Anspruch nehmen möchte, muß sie noch vor Beginn der Rentenzahlungen in Pensionsansprüche umwandeln lassen. ${ }^{88}$ Dieses Prinzip wurde mit der Einführung von nuvos abgeschafft. Die Zahlung einer Pauschale findet unter nuvos nur noch nach einer Umwandlung von Pensionsansprüchen statt. Im Jahr 2007 wurden unter dem PCSPS für jeden pensionierten civil servant durchschnittlich $£ 7.262$ an Leistungen (sowohl in Gestalt von Pauschalzahlungen als auch von Rentenzahlungen) ausbezahlt. ${ }^{89}$

Zur Finanzierung von nuvos tragen die civil servants mit eigenen Beiträgen in Höhe von 3,5\% des pensionsrelevanten Verdienstes bei. Die Beitragshöhe blieb im Vergleich zu premium gleich, während der Beitrag bei classic lediglich $1,5 \%$ betrug und dort primär der Finanzierung der Hinterbliebenenversorgung diente. Die vom Arbeitnehmer gezahlten Beiträge vermindern dessen zu versteuerndes Einkommen. Die Höhe der Arbeitgeberbeiträge wird so festgelegt, daß sie zusammen mit den Beiträgen der civil servants die Kosten der bislang erworbenen Rentenrechte decken. ${ }^{90}$ Die Arbeitgeberbeiträge variieren prozentual und steigen bei höheren Gehältern. Sie belaufen sich derzeit auf durchschnittlich 19,4\% des pensionsrelevanten Gehalts. ${ }^{91}$

Nuvos führt zu einem contracting-out aus S2P. Civil servants haben grundsätzlich die Möglichkeit, die betriebliche Versorgung zu verlassen (sog. opting-out) und zur S2P zurückzukehren. Hiervon wird aber praktisch kein Gebrauch gemacht. 92 Zudem bleibt nach einem opting-out die Rückkehr in das PCSPS jederzeit möglich.

Grundsätzlich gelten die Vorgaben des Pensions Act 1995 und die darin enthaltenen Regulierungsbefugnisse des Pensions Regulator auch für das PCSPS, allerdings enthält das Gesetz eine Reihe von Ausnahmen und Sondervorschriften für public service pensi-

87 Dies entspricht ca. $25 \%$ des Nominalwerts der Rente. Zu dieser Grenze siehe Principal Civil Service Pension Scheme 1974, Section III (The 2007 Section), rule E.16 para. (4) i.V.m. Finance Act 2004 (2001 Chapter 12), sections 241, 160(2), (5), 164, 166 i.V.m. Schedule 29 paras. 1, 2(5), (6).

88 Vgl. Principal Civil Service Pension Scheme 1974, Section II (The 1972 Section), rule 3.1 para. (b) i.V.m. rule 3.1a.

89 Vgl. Cabinet Office, Civil Superannuation Ressource Accounts 2006-07, 2007, S. 6, 32. Der durchschnittliche Bruttoverdienst der in Vollzeit beschäftigten civil servants betrug zu dieser Zeit $£ 22.520$, s. Office for National Statistics, Civil Service Statistics, 31 March 2008 (Fußn. 3), S. 3.

$90 \mathrm{Zu}$ Finanzierungsproblemen der betrieblichen Altersversorgungssysteme des öffentlichen Sektors vgl. Clark, The UK Occupational Pension System in Crisis, in: Pemberton/Thane/Whiteside (Hrsg.), Britain's Pension Crisis: History and Policy, 2006, S. 145, $162 \mathrm{ff}$.

$91 \mathrm{Zu}$ den Abstufungen und zum Durchschnittswert siehe Cabinet Office, Civil Superannuation (Fußn. 89), S. 8.

92 Rothenbacher, Civil (or Public) Servants in Europe (Fußn. 75), S. 48. Dies zeigt auch die Zahl von 594.000 aktiven Mitglieder von PCSPS, vgl. Cabinet Office, Civil Superannuation (Fußn. 89), S. 6. Allerdings sind neben den civil servants noch einige andere, kleinere Gruppen öffentlicher Bediensteter einbezogen, vgl. Superannuation Act 1972, Schedule 1. Vgl. auch Pensions Commission, Pensions: Challenges and Choices, 2004, S. 93 (Figure 3.40). 
on schemes. Aufgrund des auf einem Umlageverfahren basierenden Finanzierungsmodells sowie wegen des beim Civil Service ohnehin bestehenden Schutzes vor der Insolvenz des Arbeitgebers ist das PCSPS von der Sicherung durch den Pension Protection Fund ausgenommen.

\section{Private Rentenkonten}

\section{a) Personal Pensions und Stakeholder Pensions}

Bei personal pension schemes handelt es sich um persönliche ${ }^{93}$ Rentenkonten des Versicherten, die bei privaten Finanzunternehmen geführt werden und nicht an das Beschäftigungsverhältnis mit einem bestimmten Arbeitgeber anknüpfen. Sie wurden zum 1.7.1988 94 als alternatives, gesetzlich ausgestaltetes Vorsorgesystem geschaffen, sollen insbesondere die von betrieblichen Systemen ausgehenden Mobilitätshindernisse am Arbeitsmarkt überwinden ${ }^{95}$ und können seitdem unter bestimmten Voraussetzungen 96 zum contracting-out von S2P herangezogen werden. Im Falle eines contracting-out gelten besondere Vorschriften hinsichtlich des Teils, der der aufgegebenen S2P entspricht und durch Rabatte auf die National Insurance Beiträge finanziert ist (sog. ,protected rights pension"). 97

Die personal pensions stellen defined contributions schemes dar, bei denen die Höhe der Rente von den Kapitalerträgen abhängt, die das Finanzunternehmen mit den einbezahlten Beiträgen erzielt. Somit trägt bei diesen Rentenplänen der Arbeitnehmer das Risiko, ob die erwirtschaftete Rente auch tatsächlich höher ist als eine alternative S2P oder eine betriebliche Rente. Vor diesem Hintergrund wurde durch den Pensions Act 2007 die Möglichkeit geschaffen, defined contribution schemes künftig ab einem erst noch festzulegenden Zeitpunkt nicht mehr als Basis für ein contracting-out anzuerkennen. ${ }^{98}$

93 Trotz dieser persönlichen Zuordnung sind Konten im Falle einer Zahlungsunfähigkeit des Versicherten vor dem Zugriff durch Insolvenzgläubiger geschützt, vgl. Pension Schemes Act 1993 (1993 Chapter 48), section 159A.

94 Die personal pensions wurden durch den Social Security Act 1986 Chapter 50 eingeführt.

95 Hierzu Emmerson, Pension Reform in the United Kingdom: Increasing the Role of Private Provision?, in: Clark/Whiteside (Hrsg.), Pension Security in the $21^{\text {st }}$ Century, 2003, S. 168, $188 \mathrm{f}$.

96 Vgl. Pension Schemes Act 1993, sections 9 ff., 25A ff.

97 Einzelheiten finden sich etwa in der Personal and Occupational Pension Schemes (Protected Rights) Regulations 1996 (S.I. 1996 No. 1537). Die protected rights pension muß etwa bis zu einer Rate von $5 \%$ inflationsgesichert werden. $\mathrm{Zu}$ den Vorgaben vgl. auch Blake, Pension Schemes (Fußn. 43), S. 201.

98 Siehe oben, S. 220. 
Ein neuer Typus privater Rentenkonten wurde durch den Welfare Reform and Pensions Act 199999 in Gestalt sog. stakeholder pensions geschaffen. Auch bei diesen handelt es sich um defined contribution schemes, die auf Anlagen am Kapitalmarkt basieren. Arbeitgeber mit mindestens fünf Arbeitnehmern, die keine eigenen betrieblichen Altersvorsorgesysteme unterhalten, müssen ihren Arbeitnehmern ein derartiges stakeholder pension scheme zugänglich machen. Die Besonderheit bei dieser Vorsorgeform besteht darin, daß es sich um kollektive Verträge handelt, bei denen Obergrenzen für Gebühren, bestimmte vertragliche Mindestbeitragserfordernisse sowie besondere Anforderungen an die Übertragbarkeit bei einem Wechsel des Arbeitgebers bestehen. 100 Zudem berechtigen sie bislang ${ }^{101}$ zum contracting-out von S2P. Nimmt der Arbeitnehmer an einem stakeholder System teil, hat der Arbeitgeber einen Teil des Lohns des Arbeitnehmers im Sinne einer Entgeltumwandlung direkt in dieses scheme zu bezahlen. Eine Pflicht zur Leistung von eigenen Beiträgen durch den Arbeitgeber besteht dabei allerdings nicht.

Der Welfare Reform and Pensions Act 1999 enthält Vorgaben für die Ausgestaltung und Verwaltung der stakeholder pension schemes. Sie müssen entweder als Sondervermögen in Gestalt eines trust ausgestaltet sein oder auf einem Vertrag mit einem Finanzdienstleister beruhen, der seinerseits bei der Financial Services Authority registriert und den Vorschriften für Finanzdienstleistungen unterworfen ist. ${ }^{102}$ Zudem ist ein Großteil der für die personal pensions geltenden Vorschriften auf die stakeholder pensions anwendbar, im Falle eines contracting-out insbesondere auch die Sonderregelungen im Hinblick auf die protected rights Komponente. ${ }^{103}$ Die stakeholder pension schemes müssen ferner bei The Pensions Regulator registriert sein, ${ }^{104}$ dessen Aufgabe es ist, die Sicherheit der Anlagen und die ordnungsgemäße Verwaltung der Rentenfonds sicherzustellen. 105

\section{b) Partnership pension accounts}

Das stakeholder pension scheme im Civil Service trägt den Namen partnership. In partnership leistet der Arbeitgeber Beiträge zwischen $3 \%$ und 12,5\% der pensionable earnings in Abhängigkeit vom Alter des Arbeitnehmers. ${ }^{106}$ Tätigt der Arbeitnehmer auf

991999 Chapter 30.

100 Dazu im Einzelnen Blake, Pension Schemes (Fußn. 43), S. 231 ff.

$101 \mathrm{Zu}$ den Änderungen durch den Pensions Act 2007 siehe oben, S. 220.

102 Vgl. Welfare Reform and Pensions Act 1999, section 1; zum Regulierungsregime s. auch Blömeke, Regulierung (Fußn. 64), S. 82 ff., 100.

103 Näher Blake, Pension Schemes (Fußn. 43), S. 229.

104 Welfare Reform and Pensions Act 1999, section 2.

$105 \mathrm{Zu}$ den Anforderungen und zur Regulierung siehe Blake, Pension Schemes (Fußn. 43), S. 222 ff., 227 ff.; Blömeke, Regulierung (Fußn. 64), S. 106 ff.

$106 \mathrm{Zu}$ den Abstufungen im Einzelnen siehe Civil Service Pensions, partnership pension account, 2008 $<$ http://www.civilservice.gov.uk/Assets/PPA_1_080617_tcm6-1875.pdf > (Stand: 25.7.2009), S. 5. 
freiwilliger Basis eigene, steuerbegünstigte Beitragszahlungen, so hat der Arbeitgeber einen zusätzlichen Beitrag in Höhe der Beiträge des Arbeitnehmers bis zu maximal $3 \%$ der pensionable earnings zu leisten. ${ }^{107}$ Der partnership tarif führt zwar nicht automatisch zu einem contracting-out von S2P, was bedeutet, daß der Arbeitnehmer, der für partnership optiert, weiterhin auch Leistungsansprüche der S2P erwerben kann. Wird die stakeholder pension jedoch als Basis eines contracting-out aus S2P gewählt, wird zusätzlich ein Teil der National Insurance Beiträge als Rabatt vom Staat in das Konto einbezahlt. 108

Das Rentenkonto besteht aus einem oder mehreren Anlagefonds mit unterschiedlicher Risikostruktur, zwischen denen der civil servant wählen kann. Die Beiträge werden von einem pension provider ${ }^{109}$, der dem oben genannten Regulierungsregime unterstellt ist, am Kapitalmarkt investiert.

Über den erwirtschafteten Anlagebetrag kann der civil servant zu einem beliebigen Zeitpunkt zwischen Vollendung des 50. (ab 2010: 55.) und des 75. Lebensjahres verfügen. ${ }^{110}$ Er kann bis zu $25 \%$ des Guthabens als steuerfreie lump sum beziehen, ${ }^{111}$ der verbleibende Teil dient dem Erwerb einer Rente (annuity). ${ }^{112}$ Diese muß zunächst bei einem (beliebigen) Anbieter am Kapitalmarkt gekauft werden. Ob die umgewandelten Rentenansprüche an die Inflationsrate angepaßt werden, hängt von der Art der erworbenen annuity ab. ${ }^{113}$

Alternativ ist bis zur Vollendung des 75. Lebensjahres ein aufgeschobener Annuitätserwerb (,income draw down“) möglich, der es zuläßt, einen bestimmten Teil ${ }^{114}$ des Guthabens direkt - d.h. ohne Verrentung - als Einkommen zu beziehen und die eigentlichen Rentenzahlungen aufzuschieben. Diese Option ist angesichts der Tatsache, daß sich die annuity rates während des Aufschubs deutlich verschlechtern können, heftig in Kritik geraten.

Partnership kann seit dem 1.10.2002 von neu eingestellten civil servants als Alternative zum PCSPS gewählt werden. Die civil servants werden mit ihrer Einstellung grund-

107 Civil Service Pensions, partnership pension account (Fußn. 106), S. 5 f.

108 Civil Service Pensions, partnership pension account (Fußn. 106), S. 12.

109 Derzeit kann der Arbeitnehmer zwischen den Anbietern Standard Life, Scottish Widows und TUC/Prudential wählen, vgl. Civil Service Pensions, partnership pension account (Fußn. 106), S. 8.

110 Vgl. Finance Act 2004, sections 165(1), 279.

111 Welfare Reform and Pensions Act 1999, section 1(9) i.V.m. Finance Act 2004, sections 153, 158, 241, 160(2), (5), 164, 166 i.V.m. Schedule 29 paras. 1, 2(5), (6). Siehe auch Civil Service Pensions, partnership pension account (Fußn. 106), S. 4.

112 Zusätzlich zu den Altersrenten sieht partnership im Falle des krankheitsbedingten Ausscheidens aus dem Dienst zusätzlich zum bisher angesparten Betrag eine Pauschalzahlung vor. Dies gilt auch im Falle des Todes des civil servants vor Rentenantritt. Der Umfang, in dem Angehörige ab der Inanspruchnahme von Renten abgesichert werden sollen, hängt von der erworbenen annuity ab.

113 Civil Service Pensions, partnership pension account (Fußn. 106), S. 12.

114 Dieser Teil beträgt pro Jahr - vereinfacht gesprochen - zwischen 0 und $120 \%$ der Jahresrente, die zum jeweiligen Zeitpunkt aus dem Fonds gekauft werden könnte, vgl. Finance Act 2004, section 165(1) i.V.m. Schedule 28 paras. 4, 10(6), 14. 
sätzlich ${ }^{115}$ automatisch Mitglieder von nuvos, sie können aber binnen drei Monaten ab Dienstbeginn rückwirkend für partnership optieren. ${ }^{116}$ Das Civil Service Pensions Department des Cabinet Office geht davon aus, daß bei einer Laufzeit von mehr als zehn Jahren bei gleichen Beiträgen des Arbeitnehmers nuvos zu höheren Rentenzahlungen als partnership führen dürfte. ${ }^{117}$ Bis zum 31. März 2007 hatten sich jedoch lediglich weniger als $1 \%$ der civil servants für die stakeholder Option entschieden. 118

\section{c) Civil Service Additional Voluntary Contribution Scheme}

Mit dem Finance Act (No. 2) 1987119 wurde die Möglichkeit geschaffen, zusätzlich $\mathrm{zu}$ einer Mitgliedschaft in einem betrieblichen Versorgungssystem unter steuerlichen Vergünstigungen sog. free-standing additional voluntary contributions in private Rentenkonten einzuzahlen. ${ }^{120}$ Den civil servants steht hierfür das Civil Service Additional Voluntary Contribution Scheme mit Sonderkonditionen bezüglich der Gebühren zur Verfügung. ${ }^{121}$ Der Beitritt ist Mitgliedern von nuvos bzw. classic, classic plus oder premium vorbehalten, der Arbeitgeber leistet keine Beiträge.

\section{Bewertung}

Das Vereinigte Königreich verfügt über kein Berufsbeamtentum im herkömmlichen Sinne. Die hier zum Vergleich herangezogenen civil servants üben zwar funktional Tätigkeiten aus, die in anderen Staaten von Beamten übernommen werden. Ihre Stellung unterscheidet sich aber deutlich von einem beamtenrechtlichen Status.

Hinsichtlich der Altersversorgung sind die civil servants in das allgemeine, durch eine Vielzahl von Wahlmöglichkeiten gekennzeichnete ${ }^{122}$ System eingegliedert, so daß sie insbesondere die Basic State Pension als Grundsicherung beanspruchen können. Für sie besteht jedoch bereits seit den Anfängen des 19. Jahrhunderts eine umfassende zu-

115 Anders ist dies z.B. bei kurzzeitigen Anstellungen von weniger als zwölf Monaten.

116 Ein späterer Wechsel von nuvos zu partnership und ggf. wieder zurück bleibt möglich, kann jedoch insgesamt nur einmal in jede Richtung erfolgen, vgl. Civil Service Pensions, Pension Choices: nuvos or partnership, $2007<\mathrm{http}: / /$ www.civilservice.gov.uk/Assets/pc_nop_tcm6-1871.pdf> (Stand: 25.7.2009), S. 24 ff.

117 Civil Service Pensions, Pension Choices (Fußn. 116), S. 20.

118 Vgl. Cabinet Office, Civil Superannuation (Fußn. 89), S. 6.

1191987 Chapter 51.

120 Dazu Blake, Pension Schemes (Fußn. 43), S. 159 ff.

$121 \mathrm{Zu}$ den Einzelheiten s. das Civil Service Additional Voluntary Contribution Scheme vom 21.12.1988, das unter dem Superannuation Act 1972, section 1, erlassen wurde.

122 Zur daraus resultierenden Komplexität und Unübersichtlichkeit des Systems Emmerson, in: Clark/Whiteside (Hrsg.), Pension Security (Fußn. 95), S. 168, 190 f. 
sätzliche betriebliche Altersversorgung mit relativ hohen Beiträgen seitens der Arbeitgeber, die im Laufe der Zeit einigen, stets moderaten Änderungen unterzogen wurde. Mit Einführung von nuvos im Zuge der letzten Reform wurde das Abstellen auf das letzte Gehalt zur Berechnung der Rente aufgegeben und durch einen kontinuierlichen Erwerb von Rentenanteilen basierend auf der gesamten Erwerbsbiographie ersetzt. Im Gegenzug wurden jedoch die jährlichen Rentenquoten erhöht.

Bereits seit 2002 steht den civil servants in Gestalt des partnership accounts eine Anlagemöglichkeit am Kapitalmarkt unter finanzieller Beteiligung des Arbeitgebers zur Verfügung, die sie anstelle der klassischen betrieblichen Versorgung wählen können. Das partnership scheme ist in ein umfassendes Regulierungssystem zum Schutz der Einlagen eingebettet. Das PCSPS ist so ausgestaltet, daß civil servants automatisch der klassischen betrieblichen Versorgung zugeordnet sind, solange sie sich nicht ausdrücklich für partnership entscheiden. Mehr als $99 \%$ der civil servants blieben bislang in den betrieblichen defined benefit schemes. Dieser Trend dürfte sich angesichts der Tatsache, daß in näherer Zukunft money purchase schemes nicht mehr Grundlage für ein contracting-out aus S2P sein können, nicht grundlegend ändern.

Die civil servants haben die Möglichkeit, einen Teil ihrer nuvos oder partnership Rente in eine steuerfreie Pauschalzahlung umzuwandeln. Dies kann jedoch nur innerhalb bestimmter Grenzen geschehen und ist zudem nicht losgelöst von der Basic State Pension zu bewerten, die weiterhin als - wenn auch geringe - Universalrente geleistet wird und als feste Basis zu den Renten hinzutritt, die nach Abzug der lump sum aus nuvos oder partnership verbleiben. 
